\title{
Coherence and visibility for vectorial light
}

\author{
Alfredo Luis \\ Departamento de Óptica, Facultad de Ciencias Físicas, Universidad Complutense, \\ 28040 Madrid, Spain (alluis@fis.ucm.es)
}

Received May 10, 2010; accepted June 11, 2010;

posted June 15, 2010 (Doc. ID 128224); published July 6, 2010

Two-path interference of transversal vectorial waves is embedded within a larger scheme: this is four-path interference between four scalar waves. This comprises previous approaches to coherence between vectorial waves and restores the equivalence between correlation-based coherence and visibility. (c) 2010 Optical Society of America

OCIS codes: $030.1640,260.2110,260.5430$.

\section{INTRODUCTION}

Coherence is a key subject in optics derived from the statistical nature of real light beams [1,2]. Coherence may represent at least two attributes of the electromagnetic field:

(a) Correlations between the complex amplitudes of electromagnetic field modes.

(b) Ability to interfere as visibility of interference fringes.

For scalar waves these attributes coincide, and coherence means essentially maximum visibility. Moreover, maximum coherence is obtained when correlations factorize.

For vectorial light the situation is more complex [3]. Most of the work has focused on two-beam interference. In such a case coherence can be defined directly by the visibility of interference fringes disregarding polarization [4-8]. On the other hand, correlation-based degrees of coherence have also been introduced [9-11]. At difference with the scalar case, these two approaches can lead to contradictory conclusions, as illustrated by the discussions in [10-14]. Moreover, the relation between factorization of correlations and visibility is rather involved $[15,16]$.

In this work we embed two-path interference of transversal vectorial waves within a larger framework: this is four-path interference, where fringes depend on four independent phases, being in this way sensitive to all field correlations. This enlarged framework allows us to accommodate different approaches to coherence, restoring the agreement between correlation-based coherence and visibility.

In a previous work [17] we have studied very general abstract relations between coherence and fringe visibility for an arbitrary number of field components. This is particularized here to the case of four components. The main novelty of this paper is that we introduce a practical realization of the abstract definition of multiple-beam visibility considered in [17]. This allows us to prove the following results: (i) Correlation-based degrees of coherence are equivalent to fringe visibility. The degree of polarization is the maximum visibility that can be reached for a given field under deterministic unitary transformations.

(ii) Unit visibility holds exclusively for factorized fields.

This is illustrated with the example of a Mach-Zehnder interferometer. From a practical perspective, we show that different approaches to coherence correspond to impose different restrictions on the input beam splitter that lead to different relations between coherence and visibility. Moreover, the ultimate goal is to find the optimum input beam splitter leading to maximum visibility for given input beams. This is to obtain full advantage of the coherence conveyed by the field in the form of the best possible interference.

This is closely related to transformation properties under deterministic unitary linear transformations of different approaches, represented in this case by the properties of the input beam splitter. These are of large physical significance since they represent all transparent, linear, deterministic devices. They do not modify the randomness or disorder of the field preserving the amount of coherence $[3,6,8,18]$.

For the sake of completeness and to motivate the main definitions, in Section 2 we recall useful relations between visibility, coherence, and degree of polarization in the scalar case. These are generalized in Section 3 to the interference between two partially polarized transversal waves regarded as a four-path interferometer. This is used to relate field correlations and visibility embedding different approaches to coherence as particular cases of a more general framework.

\section{COHERENCE AND VISIBILITY FOR SCALAR WAVES}

Let us consider two harmonic electromagnetic scalar modes, statistically described by random, complex amplitudes $E_{1,2}$ with cross-spectral density matrix $\Gamma$ : 


$$
\boldsymbol{\Gamma}=\left(\begin{array}{cc}
\left\langle\left|E_{1}\right|^{2}\right\rangle & \left\langle E_{1} E_{2}^{*}\right\rangle \\
\left\langle E_{1}^{*} E_{2}\right\rangle & \left\langle\left|E_{2}\right|^{2}\right\rangle
\end{array}\right),
$$

where the angle brackets represent ensemble averages.

Coherence can manifest as two main phenomena: (1) interference as coherence between modes in the same polarization state, and (2) polarization as coherence between modes in orthogonal polarization states. These two manifestations are not independent, since we can always transform polarization into interference and vice versa by a simple polarization transformation of one of the modes.

\section{A. Coherence and Visibility}

In two-beam interference $E_{1,2}$ represent the complex amplitudes of two waves $\mathbf{E}_{j}=E_{j} \varepsilon$ in the same polarization state described by the complex unit vector $\varepsilon$. Coherence manifests through the modulation of the intensity of the interference, suitably normalized for the sake of convenience,

$$
I\left(\varphi_{1}, \varphi_{2}\right)=\frac{\left\langle\left|E_{1} e^{i \varphi_{1}}+E_{2} e^{i \varphi_{2}}\right|^{2}\right\rangle}{\left\langle\left|E_{1}\right|^{2}\right\rangle+\left\langle\left|E_{2}\right|^{2}\right\rangle}
$$

where $\varphi_{j}$ are the phases acquired by the waves within the interferometer and $I\left(\varphi_{1}, \varphi_{2}\right)$ actually depends just on the phase difference $\varphi_{1}-\varphi_{2}$. The standard expression for the fringe visibility is

$$
V(\mathbf{E})=\frac{I_{\max }-I_{\min }}{I_{\max }+I_{\min }}=\frac{2\left|\left\langle E_{1} E_{2}^{*}\right\rangle\right|}{\left\langle\left|E_{1}\right|^{2}\right\rangle+\left\langle\left|E_{2}\right|^{2}\right\rangle}=\frac{2 \sqrt{r}}{1+r}|\mu|,
$$

where $I_{\max \text { min }}$ are the maxima and minima of $I\left(\varphi_{1}, \varphi_{2}\right)$ when $\varphi_{1,2}$ are varied, $\mu$ is the complex second-order degree of coherence, and $r$ is the intensity ratio:

$$
\mu(\mathbf{E})=\frac{\left\langle E_{1} E_{2}^{*}\right\rangle}{\sqrt{\left\langle\left|E_{1}\right|^{2}\right\rangle\left\langle\left|E_{2}\right|^{2}\right\rangle}}, \quad r(\mathbf{E})=\frac{\left\langle\left. E_{1}\right|^{2}\right\rangle}{\left\langle\left|E_{2}\right|^{2}\right\rangle} .
$$

There is a clear proportionality between coherence $\mu$ and visibility $V$. There are useful alternative expressions leading to the same $V$. This is the matrix distance [3,19]

$$
V^{2}=2 \operatorname{tr}\left[\left(\gamma-\gamma_{u}\right)^{2}\right],
$$

where $\gamma$ is the normalized counterpart of $\boldsymbol{\Gamma}$, and $\gamma_{u}$ is the matrix obtained from $\gamma$ by removing all phase-dependent matrix elements:

$$
\gamma=\frac{1}{\operatorname{tr} \Gamma} \Gamma, \quad \gamma_{u}=\frac{1}{\left\langle\left|E_{1}\right|^{2}\right\rangle+\left\langle\left|E_{2}\right|^{2}\right\rangle}\left(\begin{array}{cc}
\left\langle\left|E_{1}\right|^{2}\right\rangle & 0 \\
0 & \left\langle\left|E_{2}\right|^{2}\right\rangle
\end{array}\right) .
$$

Moreover, $V$ can also be expressed as the distance between $I\left(\varphi_{1}, \varphi_{2}\right)$ and the uniform intensity distribution representing interference with null visibility [20]:

$$
V^{2}=\frac{1}{2 \pi^{2}} \int_{2 \pi} d \varphi_{1} d \varphi_{2}\left[I\left(\varphi_{1}, \varphi_{2}\right)-1\right]^{2} .
$$

\section{B. Coherence and Degree of Polarization}

In the polarization context $E_{1,2}$ represent the complex amplitudes of two orthogonally polarized waves $\mathbf{E}_{j}=E_{j} \varepsilon_{j}$ with $\varepsilon_{1} \cdot \varepsilon_{2}^{*}=0$. In this case coherence manifests in the polarization state and can be assessed through the degree of polarization $P$,

$$
P(\mathbf{E})=\frac{\left|\lambda_{1}-\lambda_{2}\right|}{\lambda_{1}+\lambda_{2}}=\left[1-4 \frac{\operatorname{det} \boldsymbol{\Gamma}}{(\operatorname{tr} \boldsymbol{\Gamma})^{2}}\right]^{1 / 2},
$$

where $\lambda_{1,2}$ are the eigenvalues of $\boldsymbol{\Gamma}$. Equivalently, in terms of $\mu$ and $r$ we have

$$
P(\mathbf{E})=\frac{\sqrt{(1-r)^{2}+4|\mu|^{2} r}}{1+r} .
$$

An alternative matrix-distance expression leading to the same $P$ is [3]

$$
P^{2}=2 \operatorname{tr}\left[(\gamma-\mathbf{i})^{2}\right], \quad \mathbf{i}=\frac{1}{2}\left(\begin{array}{ll}
1 & 0 \\
0 & 1
\end{array}\right),
$$

where $\mathbf{i}$ is the $2 \times 2$ normalized identity matrix representing fully unpolarized light.

A key point is that $P$ is invariant under deterministic unitary transformations $\quad \mathbf{E} \rightarrow \mathbf{A}=\mathbf{U E}, \quad \mathbf{A}^{t}=\left(A_{1}, A_{2}\right), \quad \mathbf{E}^{t}$ $=\left(E_{1}, E_{2}\right)$, and $\mathbf{U}$ being arbitrary $2 \times 2$ unitary matrices. This produces the unitary transformation of the crossspectral density matrix $\boldsymbol{\Gamma}(\mathbf{E}) \rightarrow \boldsymbol{\Gamma}(\mathbf{A})=\mathbf{U} \boldsymbol{\Gamma}(\mathbf{E}) \mathbf{U}^{\dagger}$. Since this preserves traces and eigenvalues we get $\gamma(\mathbf{E}) \rightarrow \gamma(\mathbf{A})$ $=\mathbf{U} \gamma(\mathbf{E}) \mathbf{U}^{\dagger}$ and $P(\mathbf{A})=P(\mathbf{E})$. However $V(\mathbf{A}) \neq V(\mathbf{E})$, since the diagonal form is not preserved, i.e., $\gamma_{u}(\mathbf{A})$ $\neq \mathbf{U} \gamma_{u}(\mathbf{E}) \mathbf{U}^{\dagger}$. Similarly, $\mu(\mathbf{A}) \neq \mu(\mathbf{E})$.

\section{Visibility and Degree of Polarization}

The two above scenarios are not independent and $V, P$ satisfy the equality [17]

$$
V^{2}+W^{2}=P^{2}
$$

where $W$ is the matrix distance between $\gamma_{u}$ and $\mathbf{i}$, and

$$
W^{2}(\mathbf{E})=2 \operatorname{tr}\left[\left(\gamma_{u}-\mathbf{i}\right)^{2}\right]=\left(\frac{\left\langle\left|E_{1}\right|^{2}\right\rangle-\left\langle\left|E_{2}\right|^{2}\right\rangle}{\left\langle\left|E_{1}\right|^{2}\right\rangle+\left\langle\left|E_{2}\right|^{2}\right\rangle}\right)^{2}=\left(\frac{1-r}{1+r}\right)^{2} .
$$

The relation (11) can be easily demonstrated from relation (10) by expressing the degree of polarization as $P^{2}$ $=2 \operatorname{tr}\left[\left(\gamma-\gamma_{u}+\gamma_{u}-\mathbf{i}\right)^{2}\right]$ and taking into account that $\gamma-\gamma_{u}$ and $\gamma_{u}-\mathbf{i}$ are trace-orthogonal matrices $\operatorname{tr}\left[\left(\gamma-\gamma_{u}\right)\left(\gamma_{u}-\mathbf{i}\right)\right]$ $=0$. This is to say that relation (11) is a version of the Pythagorean theorem as illustrated in Fig. 1.

This implies that $P$ is the maximum visibility $P$ $\geqslant V(\mathbf{A})$ under unitary transformations $\mathbf{E} \rightarrow \mathbf{A}=\mathbf{U E}$. Moreover, for every $\boldsymbol{\Gamma}$ there are unitary transformations such that the visibility reaches its maximum $P=V(\mathbf{A})$, which holds if and only if $W(\mathbf{A})=0$. For example this is the case

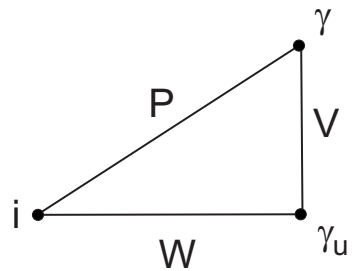

Fig. 1. Illustration of the relation $V^{2}+W^{2}=P^{2}$. 
of the transformation $\mathbf{E} \rightarrow \mathbf{A}=\mathbf{U E}$ with $\mathbf{U}=\mathbf{U}_{0} \mathbf{U}_{d}$, where $\mathbf{U}_{d}$ is the unitary matrix that diagonalizes $\boldsymbol{\Gamma}$ :

$$
\mathbf{U}_{d} \boldsymbol{\Gamma} \mathbf{U}_{d}^{\dagger}=\left(\begin{array}{cc}
\lambda_{1} & 0 \\
0 & \lambda_{2}
\end{array}\right)
$$

where $\lambda_{1,2}$ are the eigenvalues of both $\boldsymbol{\Gamma}(\mathbf{E})$ and $\boldsymbol{\Gamma}(\mathbf{A})$, and $\mathbf{U}_{0}$ is the unitary matrix

$$
\mathbf{U}_{0}=\frac{1}{\sqrt{2}}\left(\begin{array}{cc}
1 & 1 \\
1 & -1
\end{array}\right)
$$

so that

$$
\boldsymbol{\Gamma}(\mathbf{A})=\mathbf{U} \boldsymbol{\Gamma}(\mathbf{E}) \mathbf{U}^{\dagger}=\frac{1}{2}\left(\begin{array}{cc}
\lambda_{1}+\lambda_{2} & \lambda_{1}-\lambda_{2} \\
\lambda_{1}-\lambda_{2} & \lambda_{1}+\lambda_{2}
\end{array}\right),
$$

and $W(\mathbf{A})=0, V(\mathbf{A})=P$. Therefore, the practical meaning of $P$ is being maximum visibility under deterministic unitary transformations.

\section{Discussion}

Let us illustrate the above relations between visibility, polarization, and coherence by means of the Mach-Zehnder interferometer in Fig. 2. For the sake of simplicity let us assume without loss of generality that $\mu$ is real. The lossless input beam splitter $\mathbf{U}$ (with real transmission and reflection coefficients for simplicity) mixes the input complex amplitudes $E_{1,2}$ leading to the amplitudes $A_{1,2}$ for the internal modes:

$$
A_{1}=\cos \theta E_{1}+\sin \theta E_{2}, \quad A_{2}=-\sin \theta E_{1}+\cos \theta E_{2},
$$

where $\theta$ is a free parameter representing the energysplitting between modes. In the internal arms of the interferometer the modes $A_{1,2}$ experience the phase shifts $A_{1,2} \rightarrow A_{1,2} \exp \left(i \varphi_{1,2}\right)$, and then are mixed at the $50 \%$ beam splitter BS. In terms of the coherence $\mu(\mathbf{E})$ and intensity ratio $r(\mathbf{E})$ of the input beams, the visibility $V(\mathbf{A})$ of the interference at the output port of the interferometer is

$$
V(\mathbf{A})=\frac{|\sin (2 \theta)[r(\mathbf{E})-1]+2 \mu(\mathbf{E}) \sqrt{r(\mathbf{E})} \cos (2 \theta)|}{1+r(\mathbf{E})} .
$$

If visibility is a manifestation of coherence, it is legitimate to ask which is the maximum $V(\mathbf{A})$ when the input beam splitter $\mathbf{U}$ is varied, that is, when $\theta$ is varied. The maxi-

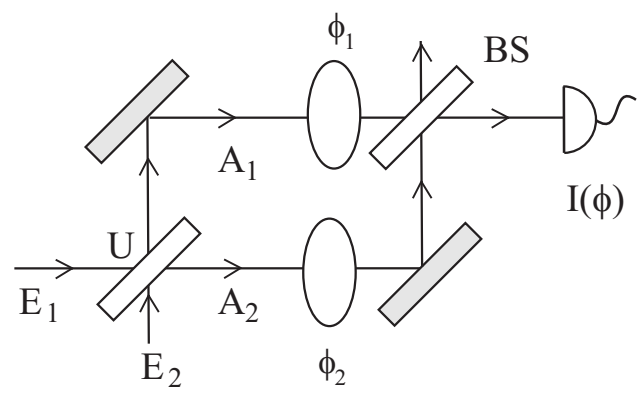

Fig. 2. Scheme of a two-path Mach-Zehnder interferometer for two scalar waves.

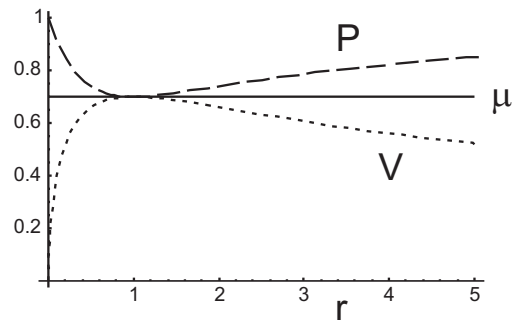

Fig. 3. Plot of $P$ (dashed curve) and $V$ (dotted curve) as functions of the intensity ratio $r$ for $\mu=0.7$ (solid curve).

mum holds for $\tan (2 \theta)=(r-1) /(2 \mu \sqrt{r})$ leading to $V_{\max }(\mathbf{A})$ $=P(\mathbf{E})$. Moreover, for the same optimum $\theta$ we get the maximum of $|\mu(\mathbf{A})|$, being $|\mu(\mathbf{A})|_{\max }=V_{\max }(\mathbf{A})=P(\mathbf{E})$. This chain of equalities holds because for the optimum $\mathbf{U}$ we get $r(\mathbf{A})=1$ and $W(\mathbf{A})=0$.

This example also allows us to emphasize that, although deeply connected, nevertheless coherence, visibility, and polarization express different ideas. This is reflected by the dependence of $P$ and $V$ on the intensity ratio $r$, while $\mu$ is independent of $r$. This is further illustrated in Fig. 3, where we have plotted $P, V$, and $\mu$ as functions of $r$ for $\mu=0.7$. They coincide when $r=1$.

\section{INTERFERENCE BETWEEN TWO TRANSVERSAL WAVES}

Let us apply the above analysis to the interference of two transversal waves with complex-amplitude vectors $\mathbf{E}_{j}^{t}$ $=\left(E_{j, x}, E_{j, y}\right), j=1,2$. Let us construct the $4 \times 4$ matrix $\boldsymbol{\Gamma}$ containing all field correlations:

$$
\boldsymbol{\Gamma}=\left(\begin{array}{ll}
\boldsymbol{\Gamma}_{1,1} & \boldsymbol{\Gamma}_{1,2} \\
\boldsymbol{\Gamma}_{2,1} & \boldsymbol{\Gamma}_{2,2}
\end{array}\right), \quad \boldsymbol{\Gamma}_{i, j}=\left(\begin{array}{cc}
\left\langle E_{i, x} E_{j, x}^{*}\right\rangle & \left\langle E_{i, x} E_{j, y}^{*}\right\rangle \\
\left\langle E_{i, y} E_{j, x}^{*}\right\rangle & \left\langle E_{i, y} E_{j, y}^{*}\right\rangle
\end{array}\right),
$$

where $\boldsymbol{\Gamma}_{1,1}$ and $\boldsymbol{\Gamma}_{2,2}$ describe polarization effects, or intrabeam correlations, and $\boldsymbol{\Gamma}_{1,2}$ represents the usual interference terms, or interbeam correlations. The fourdimensional counterparts of Eqs. (5), (10), and (12) are [17]

$$
\begin{gathered}
V^{2}(\mathbf{E})=\frac{4}{3} \operatorname{tr}\left[\left(\boldsymbol{\gamma}-\boldsymbol{\gamma}_{u}\right)^{2}\right], \quad P^{2}(\mathbf{E})=\frac{4}{3} \operatorname{tr}\left[(\boldsymbol{\gamma}-\mathbf{i})^{2}\right] \\
W^{2}(\mathbf{E})=\frac{4}{3} \operatorname{tr}\left[\left(\gamma_{u}-\mathbf{i}\right)^{2}\right],
\end{gathered}
$$

where $\gamma$ is the normalized counterpart of $\boldsymbol{\Gamma}, \mathbf{i}$ is the normalized $4 \times 4$ identity matrix,

$$
\gamma=\frac{1}{\operatorname{tr} \Gamma} \Gamma, \quad \mathbf{i}=\frac{1}{4}\left(\begin{array}{cccc}
1 & 0 & 0 & 0 \\
0 & 1 & 0 & 0 \\
0 & 0 & 1 & 0 \\
0 & 0 & 0 & 1
\end{array}\right),
$$

$\gamma_{u}$ is obtained from $\gamma$ by removing all nondiagonal matrix elements, 


$$
\gamma_{u}=\frac{1}{\operatorname{tr} \Gamma}\left(\begin{array}{cccc}
\left\langle\left|E_{1, x}\right|^{2}\right\rangle & 0 & 0 & 0 \\
0 & \left\langle\left|E_{1, y}\right|^{2}\right\rangle & 0 & 0 \\
0 & 0 & \left\langle\left|E_{2, x}\right|^{2}\right\rangle & 0 \\
0 & 0 & 0 & \left\langle\left|E_{2, y}\right|^{2}\right\rangle
\end{array}\right),
$$

and the four-dimensional vector $\mathbf{E}$ contains the four components

$$
\mathbf{E}=\left(\begin{array}{c}
E_{1} \\
E_{2} \\
E_{3} \\
E_{4}
\end{array}\right)=\left(\begin{array}{c}
E_{1, x} \\
E_{1, y} \\
E_{2, x} \\
E_{2, y}
\end{array}\right) \text {. }
$$

$P$ can be regarded as a global measure of coherence including both intrabeam and interbeam correlations [11]. Moreover $P$ is invariant under $4 \times 4$ unitary transformations $\mathbf{A}=\mathbf{U E}$ such that $P(\mathbf{A})=P(\mathbf{E})$, but in general $V(\mathbf{A})$ $\neq V(\mathbf{E})$ and $W(\mathbf{A}) \neq W(\mathbf{E})$.

A useful alternative expression for $V$ leading to the same value as the matrix distance (19) is the corresponding generalization of Eq. (7):

$$
V^{2}(\mathbf{E})=\frac{4}{3} \frac{1}{(2 \pi)^{4}} \int_{2 \pi} d \boldsymbol{\varphi}[I(\boldsymbol{\varphi})-1]^{2}=\frac{4}{3} \frac{\sum_{j \neq k}\left|\left\langle E_{j} E_{k}^{*}\right\rangle\right|^{2}}{\left(\sum_{\ell}\left\langle\left|E_{\ell}\right|^{2}\right\rangle\right)^{2}},
$$

where $\varphi=\left(\varphi_{1, x}, \varphi_{1, y}, \varphi_{2, x}, \varphi_{2, y}\right)$ are four independent phases, $d \varphi=d \varphi_{1, x} d \varphi_{1, y} d \varphi_{2, x} d \varphi_{2, y}$, and $I(\varphi)$ is the normalized intensity distribution

$$
I(\boldsymbol{\varphi})=\frac{\left\langle\left|\sum_{j, k} E_{j, k} e^{i \varphi_{j, k}}\right|^{2}\right\rangle}{\sum_{\ell, m}\left\langle\left|E_{\ell, m}\right|^{2}\right\rangle} .
$$

In Fig. 4 we have schematized a practical method to obtain the intensity distribution $I(\varphi)$ in a four-path interferometer. We have considered the Mach-Zehnder arrangement, although the same conclusions can be obtained using other configurations. The scheme is essentially a vectorial replica of the scalar case in Fig. 2. The input lossless beam splitter $\mathbf{U}$ mixes the four input complex amplitudes $\mathbf{E}_{1,2}$ leading to the amplitudes $\mathbf{A}_{1,2}$ for the internal modes.

Variable independent phase shifts $\varphi_{j, k}$ modify the phase of the complex amplitudes $A_{j, k} \rightarrow A_{j, k} \exp \left(i \varphi_{j, k}\right)$. Afterwards, there are two linear polarizers $p_{1,2}$ with axes oriented at $\pi / 4$ with respect to axes $x, y$ in the transversal

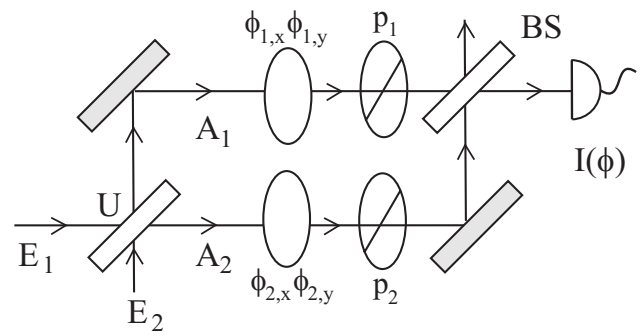

Fig. 4. Scheme of a four-path Mach-Zehnder interferometer for two vectorial waves. plane. The axes $x, y$ are chosen so that $A_{j, k} \neq 0$. Additional phases and polarization changes produced at the mirrors are embodied in the input beam splitter $\mathbf{U}$.

Finally, the beams interfere after the $50 \%$ beam splitter BS insensitive to polarization. The record of the light intensity at the detector for varying $\varphi_{j, k}$ gives $I(\varphi)$ proportional to Eq. (24), replacing $E_{j, k}$ by $A_{j, k}$. Then the visibility $V(\mathbf{A})$ can be obtained via the distance in Eq.(23). This is a four-path interferometer since the intensity distribution $I(\varphi)$ depends on four independent phase variables.

Note that the variable phase shifts $\varphi_{j, k}$ are introduced before the polarizers, so that the polarizers do not remove critical information from the field. Their role is just to mix the $x, y$ components. This simple scheme mimics the abstract projection on phase states analyzed in the quantum perspective of multi-beam coherence in [17].

As in the scalar case, the goal addressed within this scheme is to find the input beam splitter $\mathbf{U}$ that leads to the maximum visibility $V(\mathbf{A})$. That is to say, how to make the better use of the coherence conveyed by the input fields E. A different optimization procedure can be found in [21].

The Pythagorean theorem (11) holds so that $P \geqslant V$. The maximum $P=V(\mathbf{A})$ is obtained if and only if $W(\mathbf{A})=0$. The maximum visibility can always be reached by the input beam splitter $\mathbf{U}$ with $\mathbf{U}=\mathbf{U}_{0} \mathbf{U}_{d}$, where $\mathbf{U}_{d}$ is the unitary matrix that diagonalizes $\Gamma$, and

$$
\mathbf{U}_{0}=\frac{1}{2}\left(\begin{array}{cccc}
1 & 1 & 1 & 1 \\
1 & 1 & -1 & -1 \\
1 & -1 & 1 & -1 \\
1 & -1 & -1 & 1
\end{array}\right)
$$

The transformation $\mathbf{U}_{0}$ applied to the diagonal form of $\boldsymbol{\Gamma}$ produces a matrix where all elements of the diagonal are equal so that $\gamma_{u}=\mathbf{i}, W(\mathbf{A})=0$, and $V(\mathbf{A})=P$. The conclusion is that the correlation-based measure of coherence $P$ is the maximum visibility that can be achieved in four-path interference. This is exactly the same result that holds in two-beam interference between scalar waves.

\section{A. Maximum Visibility and Factorization}

We recall that $P=1$ holds if and only if there is field factorization $\left\langle E_{j} E_{k}^{*}\right\rangle=\mathcal{E}_{j} \mathcal{E}_{k}^{*}$ [11], where we are using the arrangement of components in Eq. (22). This is because

$$
P^{2}(\mathbf{E})=\frac{4}{3}\left[\frac{\operatorname{tr}\left(\boldsymbol{\Gamma}^{2}\right)}{(\operatorname{tr} \boldsymbol{\Gamma})^{2}}-\frac{1}{4}\right]=\frac{4}{3}\left[\frac{\sum_{j} \lambda_{j}^{2}}{\left(\sum_{k} \lambda_{k}\right)^{2}}-\frac{1}{4}\right],
$$

where $\lambda_{j} \geqslant 0$ are the eigenvalues of $\Gamma$. Thus, the maximum $P=1$ is obtained when $\Gamma$ has only one nonvanishing eigenvalue, so that

$$
\boldsymbol{\Gamma}=\mathbf{U}_{d}^{\dagger}\left(\begin{array}{cccc}
\lambda & 0 & 0 & 0 \\
0 & 0 & 0 & 0 \\
0 & 0 & 0 & 0 \\
0 & 0 & 0 & 0
\end{array}\right) \mathbf{U}_{d}
$$

where $\mathbf{U}_{d}$ is the unitary matrix that digonalizes $\boldsymbol{\Gamma}$. Therefore, 


$$
\left\langle E_{j} E_{k}^{*}\right\rangle=\lambda U_{d ; 1, j}^{*} U_{d ; 1, k},
$$

so that factorization holds with $\mathcal{E}_{j}=\sqrt{\lambda} U_{d ; 1, j}^{*}$.

This guarantees that unit visibility in four-path interference holds exclusively for factorized fields, as in the scalar case.

\section{B. Visibility and Interbeam Coherence}

The same four-path scheme can be used to produce interference fringes with visibility depending exclusively on the interbeam correlations in $\boldsymbol{\Gamma}_{1,2}$ in relations (18) in the form of the degree of coherence $\mu_{T S F}$ introduced in [10]. To this end, the input beam splitter $\mathbf{U}$ is of the form $\mathbf{U}=\mathbf{U}_{1}$ $\otimes \mathbf{U}_{2}$, so that the two beams $\mathbf{E}_{1,2}$ are not mixed; only their polarization is changed $\mathbf{A}_{j}=\mathbf{U}_{j} \mathbf{E}_{j}$. The transformations $\mathbf{U}_{j}$ are devised so that the intrabeam correlations $\boldsymbol{\Gamma}_{j, j}(\mathbf{A})$ in relations (18) are both diagonal in the basis of linear polarization along the $x, y$ axes. This removes from $V$ all intrabeam correlations $\left\langle A_{j, x} A_{j, y}^{*}\right\rangle=0$, so that from Eq. (23) we get

$$
V^{2}=\frac{4}{3} \frac{\sum_{j, k}\left|\left\langle A_{1, j} A_{2, k}^{*}\right\rangle\right|^{2}}{\left(\sum_{\ell, m}\left\langle\left|A_{\ell, m}\right|^{2}\right\rangle\right)^{2}}=\frac{4}{3} \frac{\operatorname{tr}\left(\boldsymbol{\Gamma}_{1,2} \boldsymbol{\Gamma}_{2,1}\right)}{\left(\operatorname{tr} \boldsymbol{\Gamma}_{1,1}+\operatorname{tr} \boldsymbol{\Gamma}_{2,2}\right)^{2}},
$$

which can be expressed in terms of $\mu_{T S F}$ [10] as

$$
V^{2}=\frac{4}{3} \frac{\operatorname{tr} \boldsymbol{\Gamma}_{1,1} \operatorname{tr} \boldsymbol{\Gamma}_{2,2}}{\left(\operatorname{tr} \boldsymbol{\Gamma}_{1,1}+\operatorname{tr} \boldsymbol{\Gamma}_{2,2}\right)^{2}} \mu_{T S F}^{2}, \quad \mu_{T S F}^{2}=\frac{\operatorname{tr}\left(\boldsymbol{\Gamma}_{1,2} \boldsymbol{\Gamma}_{2,1}\right)}{\operatorname{tr} \boldsymbol{\Gamma}_{1,1} \operatorname{tr} \boldsymbol{\Gamma}_{2,2}} .
$$

Other practical schemes relating $P$ and $\mu_{T S F}$ with measurable quantities can be found in [22-24].

\section{Two-Beam Interference Disregarding Polarization}

Finally, the coherence approaches in $[4,5]$ can be recovered within this approach with a trivial input beam splitter $\mathbf{U}=$ identity (that is, no beam mixing nor polarization changes), removing the polarizers, and adjusting the phases $\varphi_{j, x}=\varphi_{j, y}=\varphi_{j}$, so that

$$
\begin{aligned}
I\left(\varphi_{1}, \varphi_{2}\right) & \propto\left\langle\left|\mathbf{E}_{1} e^{i \varphi_{1}}+\mathbf{E}_{2} e^{i \varphi_{2}}\right|^{2}\right\rangle \\
& =\left\langle\left|\mathbf{E}_{1}\right|^{2}\right\rangle+\left\langle\left|\mathbf{E}_{2}\right|^{2}\right\rangle+2\left|\left\langle\mathbf{E}_{1}^{\dagger} \cdot \mathbf{E}_{2}\right\rangle\right| \cos \left(\varphi_{2}-\varphi_{1}+\delta\right),
\end{aligned}
$$

where $\delta$ is the argument of $\left\langle\mathbf{E}_{1}^{\dagger} \cdot \mathbf{E}_{2}\right\rangle$. Using Eq. (3) and taking into account that

$$
\boldsymbol{\Gamma}_{j, k}=\left\langle\mathbf{E}_{j} \mathbf{E}_{k}^{\dagger}\right\rangle, \quad \operatorname{tr} \boldsymbol{\Gamma}_{j, k}=\left\langle\mathbf{E}_{k}^{\dagger} \cdot \mathbf{E}_{j}\right\rangle,
$$

we get that the visibility is determined by the degree of coherence introduced in $[4,5]$

$$
V=\frac{2 \sqrt{\operatorname{tr} \boldsymbol{\Gamma}_{1,1} \operatorname{tr} \boldsymbol{\Gamma}_{2,2}}}{\operatorname{tr} \boldsymbol{\Gamma}_{1,1}+\operatorname{tr} \boldsymbol{\Gamma}_{2,2}}\left|\mu_{K W}\right|, \quad \mu_{K W}=\frac{\operatorname{tr} \boldsymbol{\Gamma}_{1,2}}{\sqrt{\operatorname{tr} \boldsymbol{\Gamma}_{1,1} \operatorname{tr} \boldsymbol{\Gamma}_{2,2}}} .
$$

\section{CONCLUSIONS}

In the above sections we have embedded different approaches to the coherence of vectorial waves within a single practical framework. This is achieved by connect- ing correlation-based degrees of polarization and coherence with the visibility of interference fringes in a fourpath interferometer.

Different approaches to coherence focus on different aspects of this field property, in the same spirit that the degrees of polarization, coherence, and fringe visibility provide complementary perspectives to the coherence of two scalar waves. From the point of view of this manuscript, different approaches to coherence determine the visibility of different interferometers. This is a practical consequence of their different symmetry properties.

In particular we have shown that the degree of polarization is equivalent to maximum visibility, as it holds in the scalar case. Moreover, we have shown also that unit visibility holds exclusively for factorized fields, as in the scalar case.

\section{ACKNOWLEDGMENTS}

This work has been supported by project No. FIS200801267of the Spanish Dirección General de Investigación del Ministerio de Ciencia e Innovación, and by project QUITEMAD S2009-ESP-1594of the Consejería de Educación de la Comunidad de Madrid.

\section{REFERENCES}

1. L. Mandel and E. Wolf, Optical Coherence and Quantum Optics (Cambridge Univ. Press, 1995).

2. J. W. Goodman, Statistical Optics (Wiley, 1985).

3. A. Luis, An Overview of Coherence and Polarization Properties for Multicomponent Electromagnetic Waves (SPIE, 2008), pp. 171-188.

4. B. Karczewski, "Degree of coherence of the electromagnetic field," Phys. Lett. 5, 191-192 (1963).

5. E. Wolf, "Unified theory of coherence and polarization of random electromagnetic beams," Phys. Lett. A 312, 263267 (2003).

6. F. Gori, M. Santarsiero, and R. Borghi, "Maximizing Young's fringe visibility through reversible optical transformations," Opt. Lett. 32, 588-590 (2007).

7. P. Réfrégier and F. Goudail, "Invariant degrees of coherence of partially polarized light," Opt. Express 13, 6051-6060 (2005).

8. R. Martinez-Herrero and P. M. Mejias, "Maximum visibility under unitary transformations in two-pinhole interference for electromagnetic fields," Opt. Lett. 32, 1471-1473 (2007).

9. H. M. Ozaktas, S. Yüksel, and M. A. Kutay, "Linear algebraic theory of partial coherence: discrete fields and measures of partial coherence," J. Opt. Soc. Am. A 19, 1563$1571(2002)$

10. J. Tervo, T. Setälä, and A. T. Friberg, "Degree of coherence for electromagnetic fields," Opt. Express 11, 1137-1143 (2003).

11. A. Luis, "Degree of coherence for vectorial electromagnetic fields as the distance between correlation matrices," J. Opt. Soc. Am. A 24, 1063-1068 (2007).

12. T. Setälä, J. Tervo, and A. T. Friberg, "Complete electromagnetic coherence in the space-frequency domain," Opt. Lett. 29, 328-330 (2004).

13. E. Wolf, "Comment on 'Complete electromagnetic coherence in the space-frequency domain'," Opt. Lett. 29, 1712-1712 (2004).

14. T. Setälä, J. Tervo, and A. T. Friberg, "Reply to comment on 'Complete electromagnetic coherence in the spacefrequency domain," Opt. Lett. 29, 1713-1714 (2004). 
15. P. Réfrégier, "Mean-square coherent light," Opt. Lett. 33, 1551-1553 (2008).

16. R. Martinez-Herrero and P. M. Mejias, "Maximizing Young's fringe visibility under unitary transformations for meansquare coherent light," Opt. Express 17, 603-610 (2009).

17. A. Luis, "Quantum-classical correspondence for visibility, coherence, and relative phase for multidimensional systems," Phys. Rev. A 78, 025802 (2008).

18. P. Réfrégier and A. Luis, "Irreversible effects of random unitary transformations on coherence properties of partially polarized electromagnetic fields," J. Opt. Soc. Am. A 25, 2749-2757 (2008).

19. F. Herbut, "A quantum measure of coherence and incompatibility," J. Phys. A 38, 2959-2974 (2005).
20. A. Luis, "Visibility for anharmonic fringes," J. Phys. A: Math. Gen. 35, 8805-8815 (2002).

21. A. Luis, "Maximum visibility in interferometers illuminated by vectorial waves," Opt. Lett. 32, 2191-2193 (2007).

22. T. Setälä, J. Tervo, and A. T. Friberg, "Contrasts of Stokes parameters in Young's interference experiment and electromagnetic degree of coherence," Opt. Lett. 31, 2669-2671 (2006).

23. T. Setälä, J. Tervo, and A. T. Friberg, "Stokes parameters and polarization contrasts in Young's interference experiment," Opt. Lett. 31, 2208-2210 (2006).

24. A. Luis, "Polarization ray picture of coherence for vectorial electromagnetic waves," Phys. Rev. A 76, 043827 (2007). 